\title{
TWO GRADIENT PROPERTIES OF EXPLICITLY CONVEX FUNCTIONS
}

\author{
XINMIN YANG
}

(Received 20 July 1992; revised 4 October 1992)

Communicated by B. Mond

\begin{abstract}
In this paper, two gradient properties of explicit convex functions are given.

1991 Mathematics subject classification (Amer. Math. Soc.): 26B25, 52A41.

Keywords and phrases: explicitly convex function, differentiable, mean-value theorem.
\end{abstract}

\section{Introduction}

Convexity plays a central role in mathematical economics, engineering, management science and optimization theory, and the subject is one currently being discussed in the mathematical programming literature, $[1,3,4]$.

The definition of explicitly convex functions appears in [5].

DEFINITION 1.1. Let $C$ be an open convex set of $R^{n}$, and $f: C \rightarrow R$. The function $f$ is said to be an explicitly convex function on $C$ if, for every pair of points $x \in C$, $y \in C, f(x) \neq f(y)$, we have

$$
f(\lambda x+(1-\lambda) y)<\lambda f(x)+(1-\lambda) f(y), \quad \forall \lambda \in(0,1) .
$$

\section{EXAMPLE 1.1. Consider}

$$
f(x)=x, \quad x \in R .
$$

Then $f$ is a convex function on $R$, but $f$ is not an explicitly convex function on $R$.

This work was supported by the National Science Foundation of China.

(C) 1995 Australian Mathematical Society 0263-6115/95 \$A2.00+0.00 
Obviously, a strictly convex function is an explicitly convex function. But the converse is not true.

EXAMPLE 1.2. This example illustrates that an explicitly convex function need not be either a convex function or a strictly convex function.

$$
f(x)=\left\{\begin{array}{lll}
1 & \text { if } & x=0 \\
0 & \text { if } & x \neq 0
\end{array}\right.
$$

Then $f$ is an explicitly convex function on $R$, but $f$ is not a convex function, nor a strictly convex function on $R$ : for $x_{1}=1$ and $x_{2}=-1, f\left(x_{1}\right)=f\left(x_{2}\right)=0$, but

$$
f\left[(1 / 2) x_{1}+(1 / 2) x_{2}\right]=f(0)=1>(1 / 2) f\left(x_{1}\right)+(1 / 2) f\left(x_{2}\right) .
$$

The following proposition shows that a local minimum of an explicitly convex function over a convex set is also a global minimum.

PROPOSITION 1.1. Let $C$ be a non-empty convex set in $R^{n}$ and $f: C \rightarrow R$. Consider the problem of minimizing $f(x)$ subject to $x \in C$. Suppose that $\bar{x} \in C$ is a local optimal solution to the problem and $f$ is an explicitly convex function. Then $\bar{x}$ is a global optimal solution.

PROOF. Since $\bar{x}$ is a local optimal solution, then there exists an $\epsilon$-neighborhood $N_{\epsilon}(\bar{x})$ around $x$ such that

$$
f(x) \geq f(\bar{x}) \text { for each } x \in C \cap N_{\epsilon}(\bar{x}) .
$$

By contradiction, suppose that $\bar{x}$ is not a global optimal solution so that $f(\hat{x})<f(\bar{x})$ for some $\hat{x} \in C$. By the explicit convexity of $f$, the following is true for each $\alpha \in(0,1)$

$$
f(\alpha \hat{x}+(1-\alpha) \bar{x})<\alpha f(\hat{x})+(1-\alpha) f(\bar{x})<f(\bar{x}) .
$$

But for $\alpha>0$ and sufficiently small, $\alpha \hat{x}+(1-\alpha) \bar{x} \in C \cap N_{\epsilon}(\bar{x})$. Hence the above inequality contradicts $\left(^{*}\right)$; this completes the proof.

From Examples 1.1 and 1.2, and Proposition 1.1, it is clear that explicitly convex functions are a very useful class and that research of such functions is worthwhile from a mathematical point of view.

In this note, based on Karamardian and Schaible's idea in [2], we give two gradient properties of explicitly convex functions. 


\section{Main results}

LEMMA 2.1. Let $C$ be a non-empty open convex set of $R^{n}$, and let $f: C \rightarrow R$ be an explicitly convex function. If $f$ is lower semi-continuous, then $f$ is a convex function on $C$.

PROOF. Let $x, y \in C$. If $f(x) \neq f(y)$, then by the definition of an explicitly convex function, we must have $f[\lambda x+(1-\lambda) y]<\lambda f(x)+(1-\lambda) f(y)$ for each $\lambda \in(0,1)$. Now suppose that $f(x)=f(y)$. To show that $f$ is a convex function, we need to show that $f[\lambda x+(1-\lambda) y] \leq f(x)$ for each $\lambda \in(0,1)$. By contradiction, suppose that $f[\alpha x+(1-\alpha) y]>f(x)$ for some $\alpha \in(0,1)$. Denote $\alpha x+(1-\alpha) y$ by $z$. Since $f$ is lower semi-continuous and explicitly convex, there exists a $\beta \in(0,1)$ such that

$$
f(z)>f[\beta x+(1-\beta) z]>f(x)=f(y) .
$$

Note that $z$ can be represented as a convex combination of $u=\beta x+(1-\beta) z$ and $y$. Hence by the explicit convexity of $f$, and since $f(u)>f(y), f(z)<f(u)$, contradicting (A). This completes the proof.

THEOREM 2.2. Let $C$ be an open convex set of $R^{n}$, and let $f: C \rightarrow R$ be a differentiable function. Then $f$ is an explicitly convex function if and only if, for every pair of points $x \in C, y \in C, f(x) \neq f(y)$, we have

$$
f(y)>f(x)+(y-x)^{T} \nabla f(x) .
$$

ProOF. Suppose that $f$ is an explicitly convex function on $C$. By Definition 1.1, for every pair of points $x \in C, y \in C, f(x) \neq f(y)$, we have

$$
f(\lambda x+(1-\lambda) y)<\lambda f(x)+(1-\lambda) f(y), \quad \forall \lambda \in(0,1) .
$$

This yields

$$
\{f[x+\lambda(y-x)]-f(x)\} / \lambda<f(y)-f(x), \quad \forall \lambda \in(0,1) .
$$

From Lemma 2.1 above and Lemma 3.1.5 in [1], we get

$$
(y-x)^{T} \nabla f(x)=\inf _{\lambda \geq 0}\{f[x+\lambda(y-x)]-f(x)\} / \lambda<f(y)-f(x),
$$

that is, $f(y)>f(x)+(y-x)^{T} \nabla f(x)$.

Conversely, suppose that for every pair of points $x \in C, y \in C, f(x) \neq f(y)$, we have

$$
f(y)>f(x)+(y-x)^{T} \nabla f(x) .
$$


Now let $z_{\alpha}=\alpha x+(1-\alpha) y, \forall \alpha \in(0,1)$. Without loss of generality, we assume $f(x)<f(y)$. We now show that $f\left(z_{\alpha}\right) \neq f(y)$.

Assume to the contrary that

$$
f\left(z_{\alpha_{0}}\right)=f(y),
$$

for some $\alpha_{0} \in(0,1)$. Now from (1), we will show that $f\left[\lambda y+(1-\lambda) z_{\alpha_{0}}\right]=f(y)$, for any $\lambda \in(0,1)$.

Indeed, if there exists $\bar{\lambda} \in(0,1)$, such that $f\left[\bar{\lambda} y+(1-\bar{\lambda}) z_{\alpha_{0}}\right] \neq f(y)$, then:

(i) If $f\left[\bar{\lambda} y+(1-\bar{\lambda}) z_{\alpha_{0}}\right]>f(y)$, let

$$
g(\lambda)=f\left[\lambda y+(1-\lambda) z_{\alpha_{0}}\right], \quad \forall \lambda \in[0,1] .
$$

Then $g$ attains a maximum on $(0,1)$. Assume that $g$ attains its maximum at $\lambda_{0} \in(0,1)$. So $\left(y-z_{\alpha_{0}}\right)^{T} \nabla f\left[\lambda_{0} y+\left(1-\lambda_{0}\right) z_{\alpha_{0}}\right]=0$, yielding

$$
\left\{\left[\lambda_{0} y+\left(1-\lambda_{0}\right) z_{\alpha_{0}}\right]-z_{\alpha_{0}}\right\}^{T} \nabla f\left[\lambda_{0} y+\left(1-\lambda_{0}\right) z_{\alpha_{0}}\right]=0 .
$$

From (1) and (2), obtain $f\left(z_{\alpha_{0}}\right)>f\left[\lambda_{0} y+\left(1-\lambda_{0}\right) z_{\alpha_{0}}\right]$, which contradicts $g$ attaining a maximum at $\lambda_{0}$.

(ii) If $f\left[\bar{\lambda} y+(1-\bar{\lambda}) z_{\alpha_{0}}\right]<f(y)$, then since $f\left(z_{\alpha_{0}}\right)=f(y)$ and $f(x)<f(y)$, we see that $f(x)<f\left(z_{\alpha_{0}}\right)$ and $f\left[\bar{\lambda} y+(1-\bar{\lambda}) z_{\alpha_{0}}\right]<f\left(z_{\alpha_{0}}\right)$. Hence the function

$$
g(\lambda)=f\left[\lambda x+(1-\lambda)\left(\bar{\lambda} y+(1-\bar{\lambda}) z_{\alpha_{0}}\right)\right]
$$

attains a maximum on $(0,1)$. Suppose this maximum occurs at $\lambda_{0}$. Then

$$
g^{\prime}\left(\lambda_{0}\right)=\left\{x-\left[\bar{\lambda} y+(1-\bar{\lambda}) z_{\alpha_{0}}\right]\right\}^{T} \nabla f\left\{\lambda_{0} x+\left(1-\lambda_{0}\right)\left[\bar{\lambda} y+(1-\bar{\lambda}) z_{\alpha_{0}}\right]\right\}=0 .
$$

Now, $f(x)<f\left\{\lambda_{0} x+\left(1-\lambda_{0}\right)\left[\bar{\lambda} y+(1-\bar{\lambda}) z_{\alpha_{0}}\right]\right\}$ implies that

$$
\begin{aligned}
f(x)> & f\left\{\lambda_{0} x+\left(1-\lambda_{0}\right)\left[\bar{\lambda} y+(1-\bar{\lambda}) z_{\alpha_{0}}\right]\right\} \\
& +\left\{x-\lambda_{0} x-\left(1-\lambda_{0}\right)\left[\bar{\lambda} y+(1-\bar{\lambda}) z_{\alpha_{0}}\right]\right\}^{T} \\
& \nabla f\left\{\lambda_{0} x+\left(1-\lambda_{0}\right)\left[\bar{\lambda} y+(1-\bar{\lambda}) z_{\alpha_{0}}\right]\right\} \\
= & f\left\{\lambda_{0} x+\left(1-\lambda_{0}\right)\left[\bar{\lambda} y+(1-\bar{\lambda}) z_{\alpha_{0}}\right]\right\} \\
& +\left(1-\lambda_{0}\right)\left\{x-\left[\bar{\lambda} y+(1-\bar{\lambda}) z_{\alpha_{0}}\right]\right\}^{T} \\
& \nabla f\left\{\lambda_{0} x+\left(1-\lambda_{0}\right)\left[\bar{\lambda} y+(1-\bar{\lambda}) z_{\alpha_{0}}\right]\right\} \\
= & f\left\{\lambda_{0} x+\left(1-\lambda_{0}\right)\left[\bar{\lambda} y+(1-\bar{\lambda}) z_{\alpha_{0}}\right]\right\}, \quad \text { a contradiction. }
\end{aligned}
$$


Combining (i) and (ii), we have

$$
f\left[\lambda y+(1-\lambda) z_{\alpha_{0}}\right]=f(y), \quad \forall \lambda \in[0,1] .
$$

Let $h(\lambda)=f\left[\lambda y+(1-\lambda) z_{\alpha_{0}}\right]$. From (3) we get

$$
0=h^{\prime}(1)=\left(y-z_{\alpha_{0}}\right)^{T} \nabla f(y) .
$$

By the hypothesis of the theorem and (4), we obtain $f(x)>f(y)$, a contradiction. Therefore

$$
f\left(z_{\alpha}\right) \neq f(y), \quad \forall \alpha \in(0,1) .
$$

If $f\left(z_{\alpha}\right)=f(x)$ for some $\alpha \in(0,1)$, then $f\left(z_{\alpha}\right)<\alpha f(x)+(1-\alpha) f(y)$ is trivial. If $f\left(z_{\alpha}\right) \neq f(x)$ for some $\alpha \in(0,1)$, then by Hypothesis (B) we have

$$
\begin{aligned}
& f(x)>f\left(z_{\alpha}\right)+\left(x-z_{\alpha}\right)^{T} \nabla f\left(z_{\alpha}\right), \\
& f(y)>f\left(z_{\alpha}\right)+\left(y-z_{\alpha}\right)^{T} \nabla f\left(z_{\alpha}\right),
\end{aligned}
$$

in view of (5). Multiplying (6) by $\alpha$, and (7) by $(1-\alpha)$, and then adding, yields

$$
f\left(z_{\alpha}\right)<\alpha f(x)+(1-\alpha) f(y) .
$$

This completes the proof of Theorem 2.2.

THEOREM 2.3. Let $f$ be differentiable on an open convex subset $C$ of $R^{n}$. Then $f$ is explicitly convex on $C$ if and only if for every pair of points $x \in C, y \in C$, $f(x) \neq f(y)$, we have

$$
(y-x)^{T}[\nabla f(y)-\nabla f(x)]>0 .
$$

ProOF. Suppose that $f$ is an explicitly convex function on $C$. Let $x, y \in C$, $f(x) \neq f(y)$. From Theorem 2.2 we have

$$
\begin{aligned}
& f(y)>f(x)+(y-x)^{T} \nabla f(x), \\
& f(x)>f(y)+(x-y)^{T} \nabla f(y) .
\end{aligned}
$$

Adding these we obtain $(y-x)^{T}[\nabla f(y)-\nabla f(x)]>0$.

Conversely, suppose that for every pair of points $x, y \in C, f(x) \neq f(y)$, we have

$$
(y-x)^{T}[\nabla f(y)-\nabla f(x)]>0 .
$$

From the mean-value theorem we obtain

$$
f(y)-f(x)=(y-x)^{T} \nabla f(\bar{x}),
$$


where

$$
\bar{x}=\lambda x+(1-\lambda) y
$$

for some $0<\lambda<1$.

(I) If $f(x) \neq f(\bar{x})$, then from (C) we obtain $(\bar{x}-x)^{T}[\nabla f(\bar{x})-\nabla f(x)]>0$. This yields

$$
(y-x)^{T} \nabla f(\bar{x})>(y-x)^{T} \nabla f(x),
$$

in view of (11). Now from (10) and (12), we have

$$
f(y)>f(x)+(y-x)^{T} \nabla f(x) .
$$

(II) If $f(x)=f(\bar{x})$, we want to show that $f(x)=f(\bar{x}) \neq f(u)$, where $u=$ $\alpha x+(1-\alpha) \bar{x}$, for some $0<\alpha<1$.

Assume to the contrary that

$$
f(x)=f(\bar{x})=f(u),
$$

where $u=\alpha x+(1-\alpha) \bar{x}$, for any $0<\alpha<1$.

$$
\text { Let } \quad \phi(\alpha)=f[x+\alpha(\bar{x}-x)], \quad \forall \alpha \in[0,1] .
$$

Then (13) implies that

$$
\phi(\alpha)=\text { const }=f(\bar{x}), \quad \forall \alpha \in[0,1]
$$

This yields

$$
0=\phi^{\prime}(1)=(\bar{x}-x)^{T} \nabla f(\bar{x})=(1-\lambda)(y-x)^{T} \nabla f(\bar{x}) .
$$

Hence, $(y-x)^{T} \nabla f(\bar{x})=0$, which together with (10) contradicts $f(x) \neq f(y)$. Thus

$$
f(x)=f(\bar{x}) \neq f(u)
$$

where

$$
u=\alpha x+(1-\alpha) \bar{x}
$$

for some $0<\alpha<1$.

Now from (B) and (15) we have

$$
(\bar{x}-u)^{T}[\nabla f(\bar{x})-\nabla f(u)]>0, \quad(x-u)^{T}[\nabla f(x)-\nabla f(u)]>0 .
$$

This yields

$$
\begin{aligned}
& (\bar{x}-x)^{T}[\nabla f(\bar{x})-\nabla f(u)]>0, \\
& (x-\bar{x})^{T}[\nabla f(x)-\nabla f(u)]>0,
\end{aligned}
$$


in view of (15). Now (16) and (17) together imply

$$
(\bar{x}-x)^{T}[\nabla f(\bar{x})-\nabla f(x)]>0 .
$$

Multiplying (18) by $1 /(1-\lambda)$ and noting (11), we obtain

$$
(y-x)^{T}[\nabla f(\bar{x})-\nabla f(x)]>0 .
$$

That is,

$$
(y-x)^{T} \nabla f(\bar{x})>(y-x)^{T} \nabla f(x) .
$$

Combining (10) and (20) we have $f(y)>f(x)+(y-x)^{T} \nabla f(x)$. Given (I) and (II), this implies that for every pair of points $x, y \in C, f(x) \neq f(y)$, we have

$$
f(y)>f(x)+(y-x)^{T} \nabla f(x) .
$$

From Theorem 2.2 we conclude that $f$ is an explicitly convex function on $C$.

\section{Acknowledgement}

The author wishes to thank the referee for several valuable suggestions which improved the presentation of this paper.

\section{References}

[1] M. S. Bazaraa and C. M. Shetty, Nonlinear programming: Theory and algorithms (Wiley, New York, 1979).

[2] S. Karamardian and S. Schaible, 'Seven kinds of monotone maps', J. Optim. Theory Appl. 66 (1990), $37-46$.

[3] A. W. Roberts and D. E. Varberg, Convex functions (New York, 1973).

[4] R. T. Rockafellar, Convex analysis (Princeton University Press, Princeton, 1970).

[5] S. Xue and S. Shen, 'Eleven kinds of convexity', Chinese Journal Oper. Res. 8 (1989), 72-75.

Department of Mathematics

Chongqing Normal University

Chongqing 630047

China 